\title{
Modulation of miR-122 expression affects the interferon response in human hepatoma cells
}

\author{
AIMEI LI ${ }^{1,2}$, JUN QIAN $^{1,2}$, JUNMING HE $^{1,2}$, QINGMENG ZHANG ${ }^{1,2}$, AIXIA ZHAI $^{1,2}$, \\ WUQI SONG ${ }^{1-3}$, YUJUN LI ${ }^{1-3}$, HONG LING ${ }^{1,2}$, ZHAOHUA ZHONG ${ }^{1,2}$ and FENGMIN ZHANG ${ }^{1-3}$ \\ ${ }^{1}$ Department of Microbiology; ${ }^{2}$ Heilongjiang Key Laboratory of Immunity and Infection; \\ ${ }^{3}$ Bio-pharmaceutical Key Laboratory, Harbin Medical University, Ministry of Education of China, \\ Harbin 150081, P.R. China
}

Received September 19, 2012; Accepted December 11, 2012

DOI: $10.3892 / \mathrm{mmr} .2012 .1233$

\begin{abstract}
Type I interferon (IFN) is believed to play significant roles in limiting tumor growth. It has been revealed that the induction of endogenous IFN expression is one of the key mechanisms for successful IFN therapy. However, recent studies have shown that the efficacy of type I IFN therapy has limitations in the clinical treatment of certain tumors, including hepatocellular carcinoma (HCC). It has been revealed that the expression of miR-122 is significantly decreased in HCC and that restoration of miR-122 expression may improve the prognosis of this condition. Previous studies also showed that patients with low miR-122 levels in the liver responded poorly to the IFN therapy. We previously identified that the IFN expression was reduced when miR-122 was suppressed in human oligodendrocytes. Based on these studies, it was hypothesized that the expression of miR-122 may modulate the endogenous IFN expression and subsequently affect the treatment outcome of IFN therapy for HCC. The results of the present study showed that miR-122-abundant Huh7 cells responded more significantly than miR-122-deficient HepG2 cells when treated with exogenous IFN. Upregulation of miR-122 significantly increased the ability of exogenous IFN-induced IFN expression, while downregulation of miR-122 decreased this ability. These data indicate that a high level of miR-122 expression may promote the expression of type I IFN induced by exogenous IFNs and further contribute to IFN therapy for HCC.
\end{abstract}

Correspondence to: Professor Fengmin Zhang, Department of Microbiology, Harbin Medical University, No. 194 Xuefu Road, Harbin 150081, P.R. China

E-mail: fengminzhang@yahoo.com.cn

Abbreviations: IFN, interferon; HCC, hepatocellular carcinoma; JAK/STAT, Janus kinase/signal transducer and activator of transcription; DMEM, Dulbecco's modified Eagle's medium; AMO-122, anti-miR-122 oligonucleotide; qRT-PCR, quantitative real time polymerase chain reaction; SEM, standard error of the mean

Key words: interferon response, miR-122, hepatoma cells

\section{Introduction}

In recent years, hepatocellular carcinoma (HCC) is one of the most common fatal malignancies worldwide (1). Whereas surgical resection is the preferred therapy, not all patients with $\mathrm{HCC}$ are suitable for this procedure and the post-operative recurrence rate remains high (2). Therefore, there is a requirement for the identification of alternative effective treatment options.

The type I interferons (IFNs) are a conserved family that play significant roles in antiviral, antitumoral and immunomodulatory functions (3). Binding of type I IFN to the IFN receptor leads to the activation of the Janus kinase/signal transducer and activator of transcription (JAK/STAT) pathway and then amplification of IFN production (4). Valuable insights into the growth-inhibitory properties of the endogenous type I IFNs have been provided from in vivo studies $(5,6)$. Although type I IFN has long been known to have antitumoral effects, it is only relatively recently that the role of type I IFN in tumor immunosurveillance has been described (7). The majority of studies have shown that IFN is able to suppress HCC growth $(8,9)$, as well as prevent its recurrence (10). However, sensitivity to type I IFN therapy in HCC patients tends to vary and the exact mechanisms remain unclear. The aim of the present study was to investigate the cellular factors that determine the sensitivity of hepatoma cells to type I IFN therapy.

\section{Materials and methods}

Cell lines. The human hepatoma cells Huh7 and HepG2 were cultured in Dulbecco's modified Eagle's medium (DMEM) supplemented with $10 \%$ fetal bovine serum and $100 \mathrm{U} / \mathrm{ml}$ penicillin/streptomycin solutions in a $37^{\circ} \mathrm{C}, 5 \% \mathrm{CO}_{2}$, humidified incubator. The cells were treated with $0.02 \%$ EDTA and $0.25 \%$ trypsin during passage.

MiR-122 plasmid and inhibitor. The plasmid pDC-316EGFP-U6-miR-122 and the normal control miRNA (miR-NC) were purchased from GeneScript (Piscataway, NJ, USA). The 2'-O-methylated, anti-miR-122 oligonucleotide (AMO-122; 5'-CAAACACCAUUGUCACACUCCA-3') and the normal control anti-miRNA (AMO-NC) were synthesized by GenePharma (Shanghai, China). 

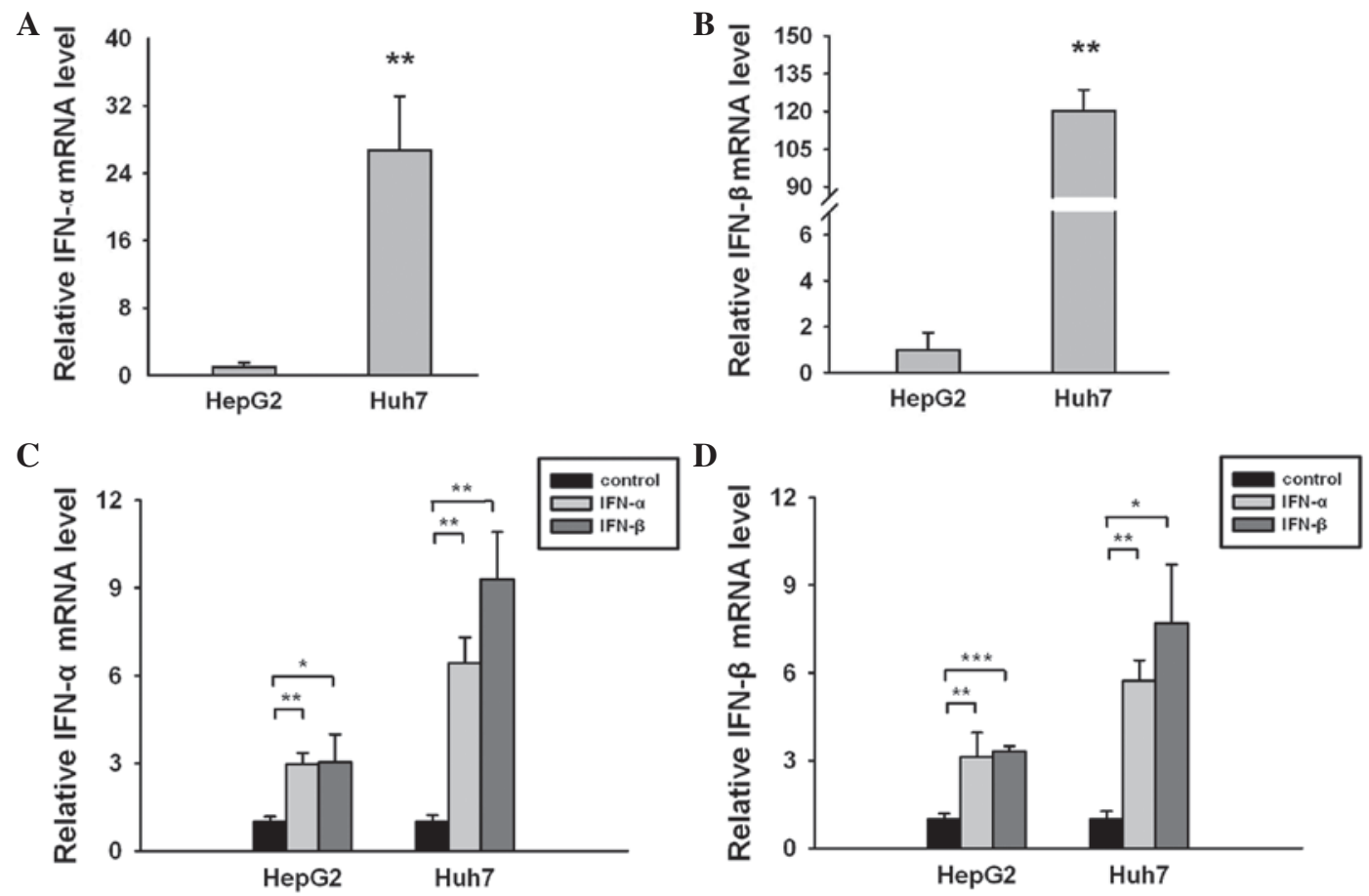

Figure 1. Expression of IFN- $\alpha / \beta$ mRNA in the HepG2 and Huh7 cells. HepG2 and Huh7 cells, cultured for 24 h, were separately incubated with exogenous IFN- $\alpha$ and IFN- $\beta(100 \mathrm{U} / \mathrm{ml})$ for $4 \mathrm{~h}$. Then total RNA was extracted for the qRT-PCR assay. (A) Basal IFN- $\alpha$ mRNA expression. (B) Basal IFN- $\beta$ mRNA expression. (C) Exogenous IFN-induced IFN- $\alpha$ mRNA expression in HepG2 and Huh7 cells. (D) Exogenous IFN-induced IFN- $\beta$ mRNA expression in HepG2 and Huh7 cells. ${ }^{*} \mathrm{P}<0.05 ;{ }^{* *} \mathrm{P}<0.01 ;{ }^{* * *} \mathrm{P}<0.001$. IFN, interferon; qRT-PCR, quantitative real time polymerase chain reaction.

Transfection of the miR-122 plasmid and inhibitor. Lipofectamine 2000 (Invitrogen, Carlsbad, CA, USA) was used to transfect the miR-122 plasmid and inhibitor into the cells. For use in the 24 -well plates, subsequent to $24 \mathrm{~h}$ culture in antibiotic-free medium, the Huh7 cells were separately transfected with $1.6 \mu \mathrm{g}$ of miR-122 plasmid or miR-NC plasmid for $48 \mathrm{~h}$ and $60 \mathrm{pmol}$ of AMO-122 or AMO-NC for $24 \mathrm{~h}$, according to the manufacturer's instructions.

Cell treatment. Human recombinant IFN- $\alpha$ and IFN- $\beta$ were purchased from Pestka Biomedical Laboratories (Piscataway, NJ, USA). Cells were incubated with $100 \mathrm{U} / \mathrm{ml}$ of IFN- $\alpha$ or IFN- $\beta$ for $4 \mathrm{~h}$ and then harvested for RNA analysis.

RNA isolation. Total RNA was extracted with TRIzol (Invitrogen) according to the manufacturer's instructions and $2 \mu 1$ total RNA was collected to measure the concentration of total RNA using a Biophotometer (Eppendorf, Hamburg, Germany). Samples with RNA absorption values of $\geq 1.9$ were considered for the study.

Determination of miR-122 and IFN- $\alpha / \beta$ RNA levels by quantitative real time polymerase chain reaction ( $q R T-P C R)$. Total RNA was incubated with specific reverse primers (for miRNAs) or oligo-dT (for mRNAs). qRT-PCR was performed to detect the miRNAs and mRNAs. Fold variations between the RNA samples were calculated subsequent to normalization to U6 RNA or GAPDH mRNA. The primers of GAPDH, IFN- $\alpha$, IFN- $\beta$ and miR-122 were described in a previous study (11).

Statistical analyses. All values are presented as the mean \pm SEM (standard error of the mean). The Student's t-test was used to evaluate the differences between two groups. All statistical tests were two-sided and $\mathrm{P}<0.05$ was considered to indicate a statistically significant difference.

\section{Results}

Differential response of the Huh7 and HepG2 cells to type I IFN stimulation. To explore the varying responses to type IIFN therapy, two common HCC cell lines, Huh7 and HepG2, were used to evaluate this phenomenon. Huh7 is miR-122-abundant while HepG2 is miR-122-deficient $(12,13)$. First, basal type I IFN expression was detected in the two cell lines. As shown in Fig. 1, the IFN expression in the Huh7 cells was significantly higher than that in the HepG2 cells. Then the two cell lines were stimulated with exogenous IFN- $\alpha$ and IFN- $\beta$ and the IFN- $\alpha / \beta$ mRNA expression in the cells treated with the exogenous IFNs was increased. In the IFN- $\alpha$ treatment, there was a 2.96(IFN- $\alpha$ mRNA) and 3.13-fold (IFN- $\beta$ mRNA) increase in the HepG2 cells, and a 6.42- (IFN- $\alpha$ mRNA) and 5.74-fold (IFN- $\beta$ mRNA) increase in the Huh7 cells. In the IFN- $\beta$ treatment, there was a 3.03- (IFN- $\alpha$ mRNA) and 3.30-fold (IFN- $\beta$ mRNA) increase in the HepG2 cells, and a 9.30- (IFN- $\alpha$ mRNA) and 7.69 -fold (IFN- $\beta$ mRNA) increase in the Huh7 cells $(\mathrm{P}<0.01)$. The increase was more pronounced in the treated Huh7 cells (Fig. 1C and D). These results confirm that the response to IFN treatment in HCC cell lines is varied, thus implying that certain factors in cells may contribute to this inconsistency.

miR-122 expression decreases in hepatoma cells following stimulation with exogenous IFNs. miR-122 has been closely associated with HCC progression (14). We previously identified that IFN expression was reduced when miR-122 was 

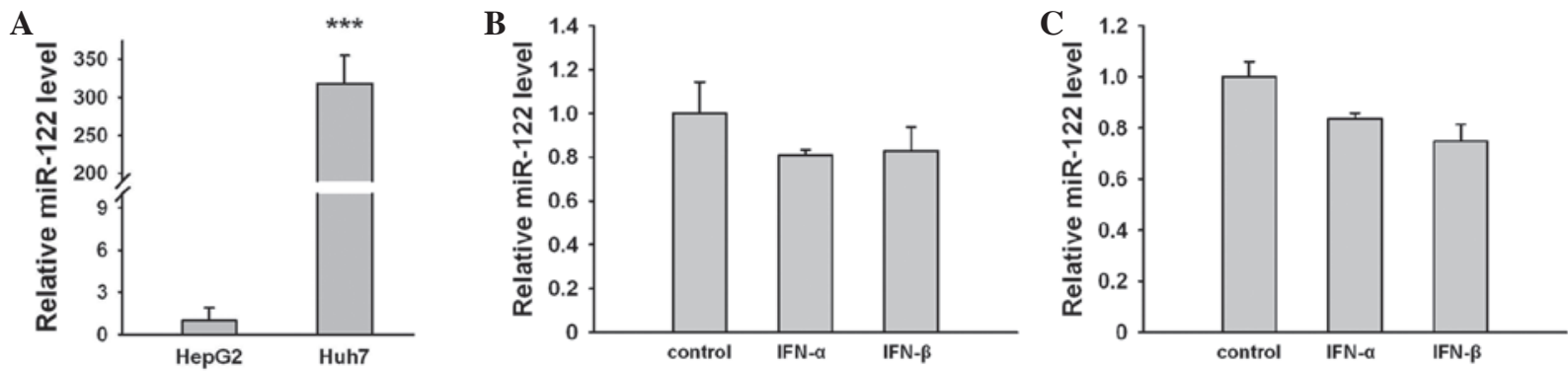

Figure 2. Exogenous IFN-induced miR-122 expression in the HepG2 and Huh7 cells. (A) Basal miR-122 expression in the HepG2 and Huh7 cells. (B) Exogenous IFN-induced miR-122 expression in the HepG2 cells. (C) Exogenous IFN-induced miR-122 expression in the Huh7 cells. ${ }^{* * *} \mathrm{P}<0.001$. INF, interferon.

A

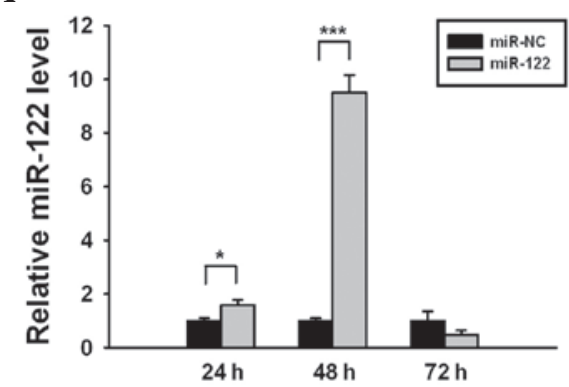

C

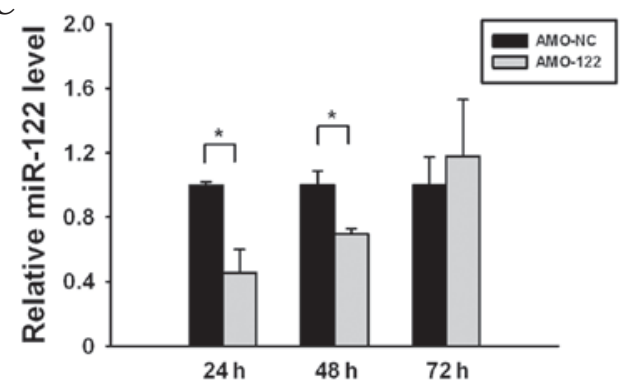

B

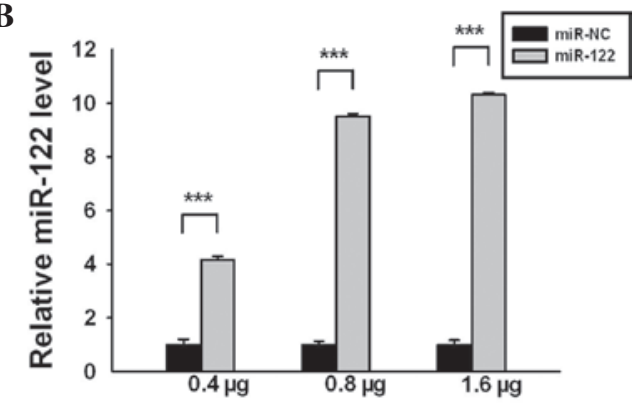

D

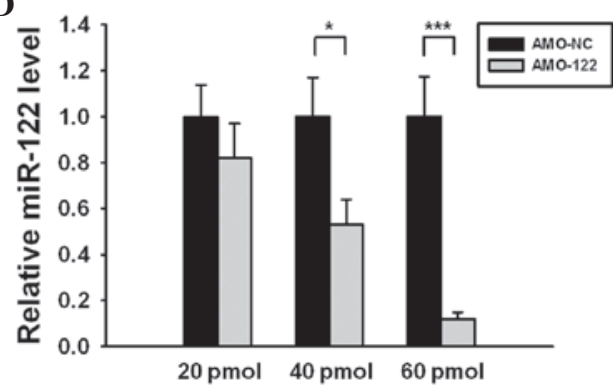

Figure 3. Verification of miR-122 plasmid and AMO-122 transfection efficiency. Subsequent to being cultured in an antibiotic-free medium for 24 h, the Huh7 cells with $\sim 70 \%$ confluency were separately transfected with the miR-122 plasmid, the miR-NC plasmid, AMO-122 and AMO-NC for various time-points and doses. The transfection was performed according to the manufacturer's instructions, and the miR-122 expression was then evaluated by qRT-PCR. The expression level of miR-122 in (A) transfection with miR-122 plasmid or the normal control miRNA (miR-NC) plasmid at 24, 48 and 72 h; (B) transfection with miR-122 plasmid or the miR-NC plasmid at 0.4, 0.8 and $1.6 \mu \mathrm{g}$; (C) transfection with AMO-122 or AMO-NC at 24, 48 and 72 h; (D) transfection with AMO-122 or AMO-NC at 20, 40 and 60 pmol. miR-NC plasmid was used as a control for the miR-122 plasmid and AMO-NC was used as a control for AMO-122. ${ }^{*} \mathrm{P}<0.05 ;{ }^{* * *} \mathrm{P}<0.001$. AMO-122, anti-miR-122; qRT-PCR, quantitative real time polymerase chain reaction; AMO-NC, normal control anti-miRNA.

suppressed in human oligodendrocytes (11). Therefore, in the present study, miR-122 expression in the two cell lines was evaluated subsequent to stimulation with exogenous IFNs. As shown in Fig. 2, the basal miR-122 expression in the Huh7 cells is markedly higher than in the HepG 2 cells. This finding is consistent with those of other related studies $(12,13)$. The present study also revealed that exogenous IFN treatment decreased the miR-122 expression in the cell lines. These data suggest that cellular levels of miR-122 may affect the IFN response.

Upregulation of miR-122 increases exogenous IFN-induced type I IFN expression. To confirm that miR-122 was involved in the regulation of the cellular response to IFN treatment, Huh7 cells (transfected with miR-122 plasmid and then incubated with exogenous IFN- $\alpha$ or IFN- $\beta$ ) were processed and analyzed for type I IFN expression by qRT-RCR. First, to get the best transfection efficiency, Huh7 cells were transfected at various time-points and with various doses of miR-122 plasmid or miR-NC plasmid. The results showed that the miR-122 levels in the Huh7 cells at 24 and 48 h post-transfection when using the miR-122-expressing plasmid were significantly higher than that of the control cells and that the highest expression was at $48 \mathrm{~h}$ (Fig. 3A). The best transfection dose for the miR-122 plasmid was identified as $1.6 \mu \mathrm{g}$ (Fig. 3B). Therefore, considering these results, $48 \mathrm{~h}$ and $1.6 \mu \mathrm{g}$ were selected for the transfection of the miR-122 plasmid in the subsequent experimental studies.

Next, the effects of miR-122 on exogenous IFN-induced type I IFN expression in the Huh7 cells were examined. qRT-PCR showed that ectopic expression of miR-122 (Fig. 4A and D) resulted in a significant increase in IFN- $\alpha / \beta$ mRNA expression following stimulation with IFN- $\alpha$ (Fig. 4B and C) or IFN- $\beta$ (Fig. 4E and F). These data reveal that miR-122 improved the IFN response mainly by increasing type I IFN expression. 
A

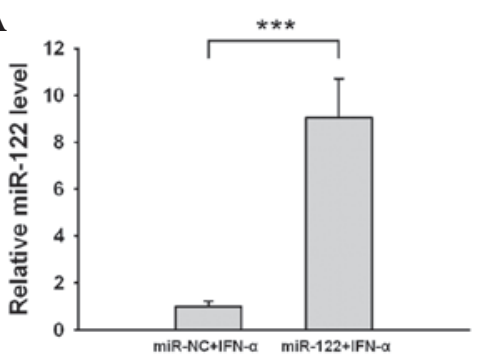

D

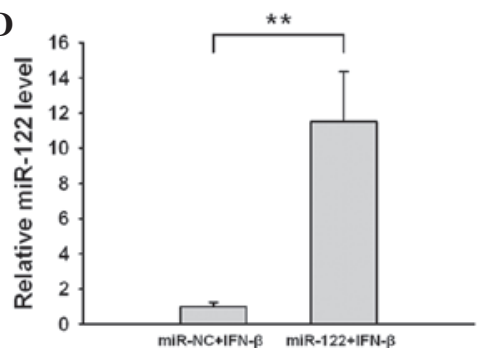

B

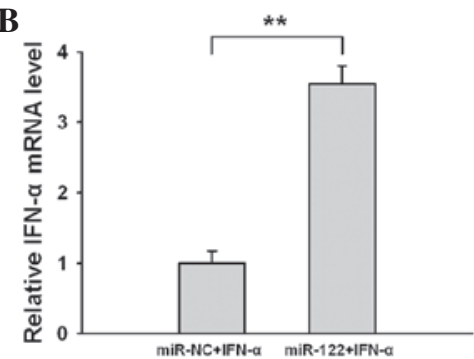

E

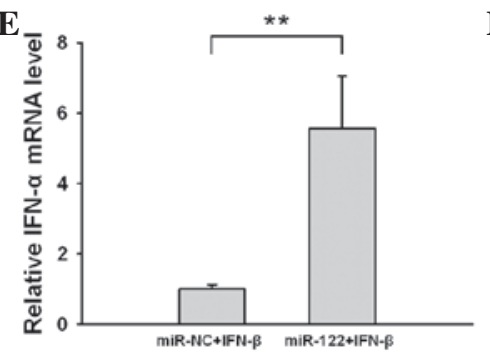

C

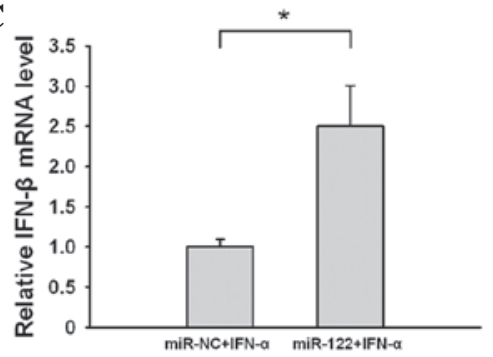

F

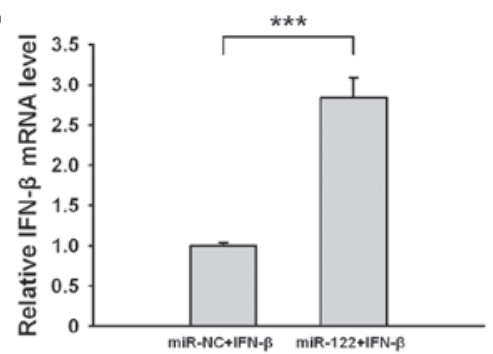

Figure 4. Effect of miR-122 upregulation on exogenous IFN-induced IFN- $\alpha / \beta$ expression. The Huh7 cells at $\sim 70 \%$ confluency were transfected with the miR-122 or miR-NC plasmid for $44 \mathrm{~h}$ and then stimulated with $100 \mathrm{U} / \mathrm{ml}$ IFN- $\alpha$ or IFN- $\beta$ for $4 \mathrm{~h}$. Total RNAs were extracted for the qRT-PCR assay. (A) miR-122 expression with exogenous IFN- $\alpha$ stimulation. (B) Exogenous IFN- $\alpha$-induced IFN- $\alpha$ mRNA expression. (C) Exogenous IFN- $\alpha$-induced IFN- $\beta$ mRNA expression. (D) miR-122 expression with exogenous IFN- $\beta$ stimulation. (E) Exogenous IFN- $\beta$-induced IFN- $\alpha$ mRNA expression. (F) Exogenous IFN- $\beta$-induced IFN- $\beta$ mRNA expression. The miR-NC plasmid was used as a control. ${ }^{*} \mathrm{P}<0.05 ;{ }^{* *} \mathrm{P}<0.01 ;{ }^{* * * *} \mathrm{P}<0.001$. IFN, interferon; miR-NC, normal control miRNA; qRT-PCR, quantitative real time polymerase chain reaction.
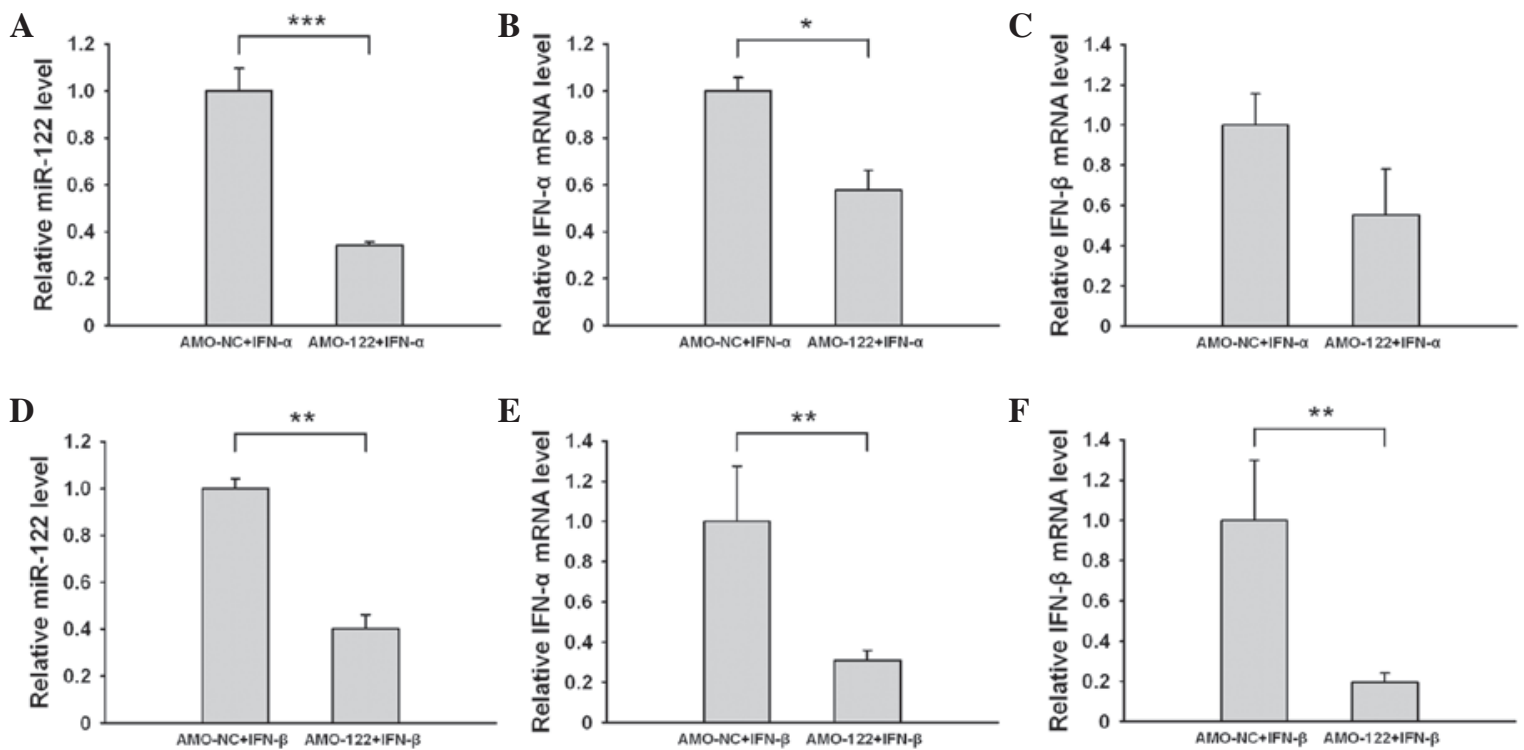

Figure 5. Effect of miR-122 downregulation on exogenous IFN-induced IFN- $\alpha / \beta$ expression. The Huh7 cells at $~ 70 \%$ confluency were transfected with AMO-122 or AMO-NC for $20 \mathrm{~h}$ and then stimulated with $100 \mathrm{U} / \mathrm{ml} \mathrm{IFN-} \alpha$ or IFN- $\beta$ for $4 \mathrm{~h}$. Total RNA was extracted for the qRT-PCR assay. (A) miR-122 expression with exogenous IFN- $\alpha$ stimulation. (B) Exogenous IFN- $\alpha$-induced IFN- $\alpha$ mRNA expression. (C) Exogenous IFN- $\alpha$-induced IFN- $\beta$ mRNA expression. (D) miR-122 expression with exogenous IFN- $\beta$ stimulation. (E) Exogenous IFN- $\beta$-induced IFN- $\alpha$ mRNA expression. (F) Exogenous IFN- $\beta$-induced IFN- $\beta$ mRNA expression. AMO-NC was used as a control. ${ }^{*} \mathrm{P}<0.05 ;{ }^{* *} \mathrm{P}<0.01 ;{ }^{* * *} \mathrm{P}<0.001$. IFN, interferon; miR-NC, normal control miRNA; AMO-122, anti-miR-122; AMO-NC, normal control anti-miRNA; qRT-PCR, quantitative real time polymerase chain reaction.

Downregulation of miR-122 decreases exogenous IFN-induced type I IFN expression. To further validate the effect of miR-122 on type I IFN expression induced by exogenous IFNs, the Huh7 cells, which were latter stimulated with

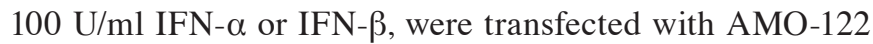
to knock down the miR-122 expression. Verification of the best transfection efficiency for AMO-122 was also sought and the results showed that AMO-122 was able to significantly suppress the miR-122 expression in the Huh7 cells at $24 \mathrm{~h}$ and 60 pmol (Fig. 3C and D). Even after exogenous IFN stimulation, IFN- $\alpha / \beta$ mRNA expression remained significantly decreased when miR-122 expression was inhibited by AMO-122 (Fig. 5). These data further confirm that the expression of miR-122 affected the exogenous IFN-induced type I IFN expression and was then involved in the IFN response. 


\section{Discussion}

Type I IFN has been associated with regulating the immune response to tumorigenesis and has therefore been widely used in the treatment of certain types of tumor $(15,16)$. HCC is one of the most frequently encountered malignant tumors in clinical practice worldwide and is associated with high mortality (1). Previous studies have shown that type I IFN may be a valuable means to inhibit the proliferation of $\operatorname{HCC}(8,9)$ and also to prevent its recurrence following surgery (10). By binding to the IFN receptor, type I IFNs activate the JAK/STAT pathway and then amplify IFN production (4). IFN has been used as an significant agent in the treatment of HCC, however, a study has reported that IFN therapy produced a partial response in certain patients with HCC (17). The mechanism behind these differing sensitivities to IFN therapy remains unclear.

In the present study, the Huh7 and HepG2 cells had varying responses to the IFN treatment. Further investigations revealed that the expression levels of cellular miR-122 may explain this inconsistency.

First the exogenous IFN function was characterized in the HCC cell lines of the present study by comparing the HepG2 and Huh7 cells. As shown in Fig. 1, the Huh7 cells expressed a markedly higher level of IFN- $\alpha / \beta$ mRNA compared with the HepG2 cells (Fig. 1A and B). The two cell lines were then separately treated with $100 \mathrm{U} / \mathrm{ml}$ IFN- $\alpha$ or IFN- $\beta$. The results showed that the IFN expression was increased in the HepG2 and Huh7 cells, but that the increase was more significant in the Huh7 cells (Fig. 1C and D). These findings suggest that the Huh7 cells had a better response to IFN treatment than the HepG 2 cells. Since the exogenous IFN- $\alpha / \beta$ proteins were used as IFN stimulators in the experiments, the supernatant was a mixture of exogenous and endogenous IFNs. Therefore, the IFN protein expression was not detected and only the IFN mRNA expression was measured by qRT-PCR assay and used to represent the IFN expression level.

Endogenous type I IFN has been identified as an essential component of the defense mechanisms to restrict tumor growth (6). In vivo studies have also provided valuable insights into the tumor growth-inhibitory property of endogenous type I IFN $(5,6)$. The dysregulation of type I IFN expression may be implicated in tumor growth, underlining the requirement to understand the host factors that contribute to IFN expression.

miRNAs are closely associated with the IFN response. Accumulated data demonstrate that miR-155 is able to enhance the immune response by an induction of IFN (18), while miR-146a (19), miR-148, miR-152 (20), miR-26a, miR-34a, miR-145 and let-7b (21) are able to effectively attenuate the IFN response. We previously identified that IFN expression was reduced when miR-122 was suppressed in human oligodendrocytes (11). Previous studies have also shown miR-122 to be significantly reduced in the HCC cell lines and the clinical HCC samples (22). A study by Coulouarn et al emphasized that miR-122 was a diagnostic and prognostic marker for HCC progression (14). Moreover, Ma et al later observed that the loss of miR-122 resulted in increased cell migration and invasion and that restoration of miR-122 levels reversed this phenotype (23). These observations emphasize the significance of miR-122 in inhibiting HCC progression. Therefore, based on all these findings, the present study sought to investigate the effect of miR-122 on the IFN response in hepatoma cells.

In the present study, cellular miR-122 levels declined with IFN treatment, but only by $10-20 \%$ (Fig. 2). This is consistent with a previous study (24). To further understand the interaction between miR-122 and IFN treatment, the effects of miR-122 overexpression and miR-122 knockdown on the exogenous IFN-induced type I IFN expression in Huh7 cells were examined. Another previous study revealed that miR-122 significantly upregulated the apoptosis of the Huh7 cells, while the HepG2 cells were not actively responsive to miR-122 due to its adaptation to the absence of miR-122 or the lack of a miR-122 target (13). The Huh7 cells were therefore used as the main model to show the alteration of type I IFN expression.

Results from the present study indicated that miR-122 overexpression significantly increased the exogenous IFN-induced IFN- $\alpha / \beta$ mRNA expression (Fig. 4), while miR-122 knockdown resulted in a decreased exogenous IFN-induced IFN- $\alpha / \beta$ mRNA expression (Fig. 5). These results further confirm that miR-122 has an significant role in exogenous IFNs-induced type I IFN expression in hepatoma cells.

In conclusion, these data suggest that cellular miR-122 levels have an effect on IFN expression and may serve as significant factors in type I IFN therapy against HCC.

\section{Acknowledgements}

The authors are grateful to Dr James Ogutu, Department of Microbiology, Harbin Medical University, China, for his critical reading of the manuscript. This study was supported by grants from the National Natural Science Foundation of China (NSFC; 30371242, 30070673, J0730858, J1103609 and 81202296).

\section{References}

1. Motola-Kuba D, Zamora-Valdes D, Uribe $M$ and Mendez-Sanchez N: Hepatocellular carcinoma. An overview. Ann Hepatol 5: 16-24, 2006.

2. Okuda K: Primary liver cancers in Japan. Cancer 45: 2663-2669, 1980.

3. Stark GR, Kerr IM, Williams BR, Silverman RH and Schreiber RD: How cells respond to interferons. Annu Rev Biochem 67: 227-264, 1998.

4. Haller O, Kochs G and Weber F: The interferon response circuit: induction and suppression by pathogenic viruses. Virology 344 : 119-130, 2006.

5. Deonarain R, Verma A, Porter AC, Gewert DR, Platanias LC and Fish EN: Critical roles for IFN-beta in lymphoid development, myelopoiesis, and tumor development: links to tumor necrosis factor alpha. Proc Natl Acad Sci USA 100: 13453-13458, 2003.

6. Gresser I and Belardelli F: Endogenous type I interferons as a defense against tumors. Cytokine Growth Factor Rev 13: 111-118, 2002.

7. Dunn GP, Bruce AT, Sheehan KC, et al: A critical function for type I interferons in cancer immunoediting. Nat Immunol 6: 722-729, 2005

8. Wang L, Tang ZY, Qin LX, et al: High-dose and long-term therapy with interferon-alfa inhibits tumor growth and recurrence in nude mice bearing human hepatocellular carcinoma xenografts with high metastatic potential. Hepatology 32: 43-48, 2000.

9. Yano H, Ogasawara S, Momosaki S, et al: Growth inhibitory effects of pegylated IFN alpha-2b on human liver cancer cells in vitro and in vivo. Liver Int 26: 964-975, 2006.

10. Lin SM, Lin CJ, Hsu CW, et al: Prospective randomized controlled study of interferon-alpha in preventing hepatocellular carcinoma recurrence after medical ablation therapy for primary tumors. Cancer 100: 376-382, 2004. 
11. Qian J, Zhai A, Kao W, et al: Modulation of miR-122 on persistently Borna disease virus infected human oligodendroglial cells. Antiviral Res 87: 249-256, 2010.

12. Jopling CL, Yi M, Lancaster AM, Lemon SM and Sarnow P: Modulation of hepatitis $\mathrm{C}$ virus RNA abundance by a liver-specific MicroRNA. Science 309: 1577-1581, 2005.

13. Wu X, Wu S, Tong L, et al: miR-122 affects the viability and apoptosis of hepatocellular carcinoma cells. Scand J Gastroenterol 44: 1332-1339, 2009.

14. Coulouarn C, Factor VM, Andersen JB, Durkin ME and Thorgeirsson SS: Loss of miR-122 expression in liver cancer correlates with suppression of the hepatic phenotype and gain of metastatic properties. Oncogene 28: 3526-3536, 2009.

15. Kirkwood J: Cancer immunotherapy: the interferon-alpha experience. Semin Oncol 29 (Suppl 7): 18-26, 2002.

16. Borden EC: Interferons - expanding therapeutic roles. N Engl J Med 326: 1491-1493, 1992.

17. Kirkwood JM, Ibrahim JG, Sondak VK, et al: High- and low-dose interferon alfa-2b in high-risk melanoma: first analysis of intergroup trial E1690/S9111/C9190. J Clin Oncol 18: 2444-2458, 2000.

18. Wang P, Hou J, Lin L, et al: Inducible microRNA-155 feedback promotes type I IFN signaling in antiviral innate immunity by targeting suppressor of cytokine signaling 1. J Immunol 185: 6226-6233, 2010.
19. Hou J, Wang P, Lin L, et al: MicroRNA-146a feedback inhibits RIG-I-dependent Type I IFN production in macrophages by targeting TRAF6, IRAK1, and IRAK2. J Immunol 183: 2150-2158, 2009

20. Liu X, Zhan Z, Xu L, et al: MicroRNA-148/152 impair innate response and antigen presentation of TLR-triggered dendritic cells by targeting CaMKII $\alpha$. J Immunol 185: 7244-7251, 2010.

21. Witwer KW, Sisk JM, Gama L and Clements JE: MicroRNA regulation of IFN-beta protein expression: rapid and sensitive modulation of the innate immune response. J Immunol 184: 2369-2376, 2010.

22. Kutay H, Bai S, Datta J, et al: Downregulation of miR-122 in the rodent and human hepatocellular carcinomas. J Cell Biochem 99: 671-678, 2006.

23. Ma L, Liu J, Shen J, et al: Expression of miR-122 mediated by adenoviral vector induces apoptosis and cell cycle arrest of cancer cells. Cancer Biol Ther 9: 554-561, 2010.

24. Sarasin-Filipowicz M, Krol J, Markiewicz I, Heim MH and Filipowicz W: Decreased levels of microRNA miR-122 in individuals with hepatitis $C$ responding poorly to interferon therapy. Nat Med 15: 31-33, 2009. 